2004

\title{
Breaking of Rotational Symmetry in Cylindrically Bounded 2D Electron Plasmas and 2D Fluids
}

Eli Sarid

Catalin Teodorescu

Philip S. Marcus

Joel Fajans

Follow this and additional works at: https://researchrepository.wvu.edu/faculty_publications

\section{Digital Commons Citation}

Sarid, Eli; Teodorescu, Catalin; Marcus, Philip S.; and Fajans, Joel, "Breaking of Rotational Symmetry in Cylindrically Bounded 2D Electron Plasmas and 2D Fluids" (2004). Faculty Scholarship. 73.

https://researchrepository.wvu.edu/faculty_publications/73 


\title{
Breaking of Rotational Symmetry in Cylindrically Bounded 2D Electron Plasmas and 2D Fluids
}

\author{
Eli Sarid, ${ }^{1,2}$ Catalin Teodorescu, ${ }^{3}$ Philip S. Marcus, ${ }^{4}$ and Joel Fajans ${ }^{1}$ \\ ${ }^{1}$ Physics Department, University of California at Berkeley, Berkeley, California 94720-7300, USA \\ ${ }^{2}$ Physics Department, NRCN, P.O. Box 9001, Beer-Sheva 84190, Israel \\ ${ }^{3}$ West Virginia University, P.O. 6315, Morgantown, West Virginia 26506-6315, USA \\ ${ }^{4}$ Mechanical Engineering, University of California at Berkeley, Berkeley, California 94720-7300, USA
}

(Received 24 April 2003; published 17 November 2004)

\begin{abstract}
Off-axis final states of cylindrically bounded 2D fluids can develop from initially unstable, but cylindrically symmetric, 2D vorticity distributions. Experiments with electrons in a MalmbergPenning trap, as well as 2D fluid simulations, demonstrated that such states result when the initial vorticity distribution is close to the boundary, while less extended distributions lead to on-axis states. A simple thermodynamic model, maximizing the entropy of a state consisting of a diffuse background surrounding a strong coherent vortex, is shown to quantitatively predict this bifurcation, while conserving circulation, angular momentum, and energy.
\end{abstract}

DOI: $10.1103 /$ PhysRevLett.93.215002

Introduction.-Two-dimensional (2D) fluids often organize spontaneously into coherent vortices. Such structures can be found in geophysical flows and planetary atmospheres; Jupiter's Great Red Spot is the best known example [1,2]. Analogies also exist between the formation of $2 \mathrm{D}$ vortices and the dynamics of galaxies and protoplanetary nebulas [3].

Equilibrium statistical mechanics of 2D incompressible ideal fluids can be used to understand the formation of coherent vorticity structures [4]. Using just a few thermodynamic variables greatly reduces the complexity of the problem compared to tracking the detailed dynamics. Several studies have employed statistical mechanics to predict the existence of off-axis equilibrium states in cylindrically bounded 2D system [5-7]. The center of mass of such states is shifted from the symmetry axis of the system. These states do not share the cylindrical symmetry of the boundary conditions, and they exist even for cylindrically symmetric initial conditions.

Here we report experiments that show, for the first time, that off-axis equilibrium states result if the initial condition is an unstable configuration with sufficiently large angular momentum. The experiments were performed with electrons in a Malmberg-Penning trap, and were corroborated with 2D fluid simulations. The electrons in these traps follow $E \times B$ drift orbits, and the drift-Poisson equations controlling their motion are isomorphic to the 2D Euler equations for an inviscid fluid. The flow vorticity is proportional to the electron density, and the electric potential is analogous to the $2 \mathrm{D}$ stream function [8]. Thus, the electrons mimic ideal twodimensional fluid equations.

We also present a simple off-axis equilibrium model that assumes that a fraction of the initial circulation evolves into a coherent vortex, while the remainder forms a diffuse background. Unlike previous models, we do not assume ergodicity. Our model shows the same quantita-
PACS numbers: 52.27.Jt, 47.20.-k, 47.32.Cc

tive behavior as the experiments and simulations. We find that the system tends to seek the maximum entropy state consistent with circulation, energy, and angular momentum conservation. For initially small radii systems (low angular momentum), the maximum entropy states are centered states. For initially large radii systems, no centered solutions are available and the system is forced to move off axis to conserve angular momentum.

Experiments and simulations.-The electron experiments were performed with the photocathode pureelectron plasma trap [9]. This trap can create nearly arbitrary, highly reproducible initial electron densityvorticity configurations. The time evolution of a particular configuration is studied by repeatedly regenerating the system and letting it evolve for ever longer times. Images of the electrons are made by dumping them onto a phosphor screen.
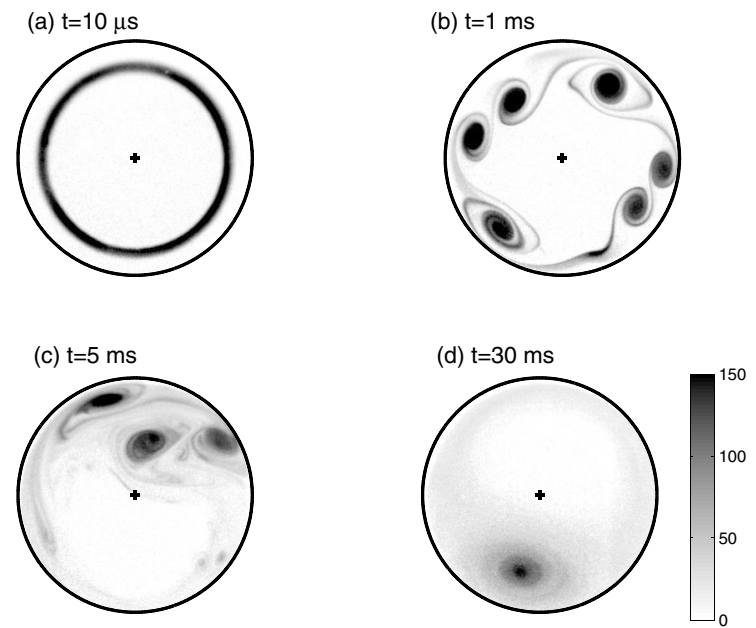

FIG. 1. Time evolution of a large ring of electrons $\left(r_{i}\right.$, the average initial radius is 0.79 ). The top of the scale (150) corresponds to a density of $6 \times 10^{6} \mathrm{~cm}^{-3}$. 
Figure 1 shows the evolution of a typical large electron ring. First, the Kelvin-Helmholtz instability breaks the ring into discrete vortices. After a short period of mixing and merging, only a single strong "vortex" remains, surrounded by an extended background. In this case, the background reaches the trap wall and has a hole. The smaller coherent vortex, with density comparable to the density of the initial ring, is shifted off axis. All this happens on a time scale short enough $(30 \mathrm{~ms})$ that the electrons behave as a $2 \mathrm{D}$ fluid. The resulting state can be regarded as a metaequilibrium because of the separation of time scales between the fast, fluidlike $E \times B$ equilibration of the electrons, and the slow, non-fluidlike exchange of energy with other degrees of freedom [6]. These nonfluid effects cause the center of charge to decay to the trap axis after several hundred milliseconds. This slow decay is likely caused by the mechanism of rotational pumping [10,11].

Figure 2 shows the evolution of a typical small electron ring. Initially, the evolution is similar to that of the larger ring: the ring breaks into discrete vortices, the vortices merge, and eventually a single strong vortex remains with an extended background. Here, however, the vortex is centered. The background is more uniform than in Fig. 1 and barely reaches the wall. For yet smaller rings, the background recedes from the wall.

The transition between initial distributions that result in on-axis states and those that result in off-axis states is quite sharp. Figure 3 shows the off-axis shift $D$ of the strong vortex as a function of the average initial radius of the ring, $r_{i}$. (The "average" radius is the densityweighted average of the distance from the trap center for the initial distribution. The wall radius is taken to equal 1.) Rings whose $r_{i}$ is less than about 0.5 produce centered final states. For larger rings, off-axis states
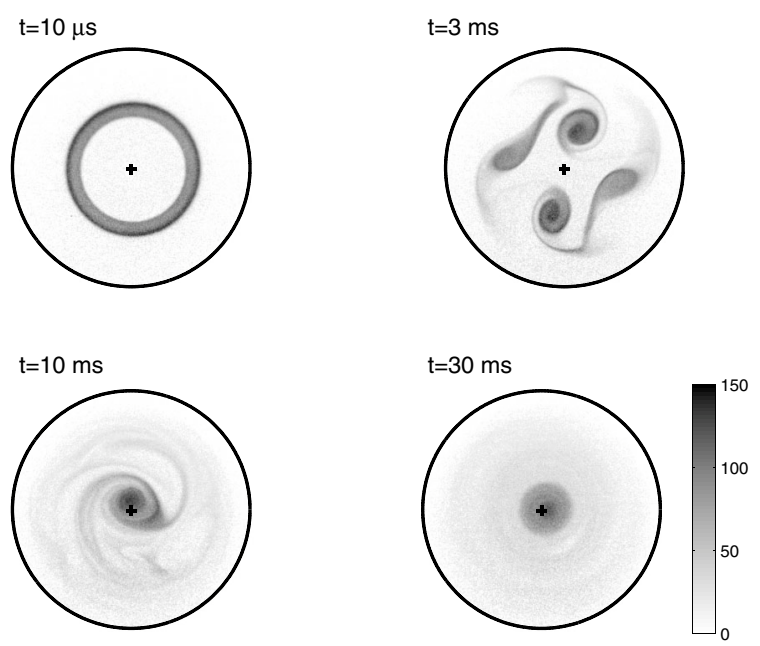

FIG. 2. Time evolution of a small ring of electrons $\left(r_{i}=0.5\right)$. The top of the scale (150) corresponds to a density of $6 \times$ $10^{6} \mathrm{~cm}^{-3}$. appear. The off-axis shift increases sharply with $r_{i}$. Moving the initial center of charge slightly off center has no significant affect on the final state.

We verified that the observed phenomena are due to $2 \mathrm{D}$ fluid dynamics by running a spectral code simulation of the Euler equation [12]. As can be seen in Fig. 3, the electron experiments and the 2D fluid simulation code agree closely; the off-axis transition is very well predicted by the code. We also find that resulting final states in the simulations typically have about half of the initial circulation in the diffuse background, and half in the small high vorticity disk. This is similar to the results of the experiments. The simulation also verifies that the off-axis states evolve with strict conservation of circulation and angular momentum. Unlike the electron experiments that inevitably include effects not described by the ideal 2D picture, the off-axis states in the 2D fluid simulations do not show a decrease of the off-axis deviation with time. The simulation also demonstrates that the results are obtained when the initial vorticity is concentrated only in the ring, with strictly zero background vorticity.

The exact geometry of the initial distribution is not crucial. We performed electron experiments with initial distributions in the shape of bars (elongated rectangles) or tribars (three rectangles merging in the center of the trap). All the initial distributions had on-axis center of charge. The initial distributions of bars and tribars broke to two and three vortices, respectively, which then merged to a single vortex. As can be seen in Fig. 4, an on-axis strong vortex resulted for the smaller initial shapes, while an off-axis state resulted for the more extended initial distributions. Figure 5 shows that the transition between on- and off-axis states is similar to that of the ring experiments. Here the results are pre-

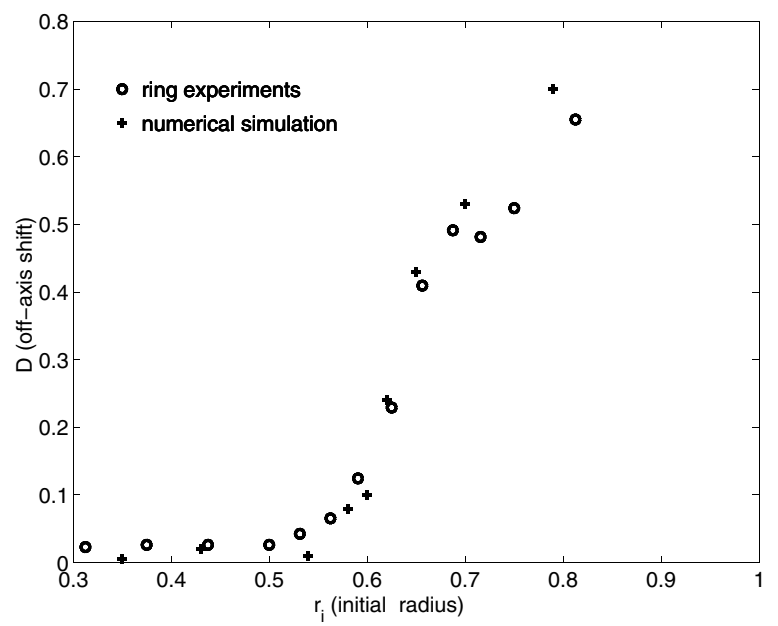

FIG. 3. Off-axis shift of the coherent strong vortex in the final state, as a function of the initial average radius of the rings. Experimental results are shown with circles, simulation results with crosses. 
sented as a function of the angular momentum. We use the same normalization as in Ref. [6], where the density $n$ is scaled such that the total circulation is $1: \int n d^{2} \mathbf{r}=1$. With this scaling, the angular momentum is just the weighted average of $r^{2}: M=\int r^{2} n d^{2} \mathbf{r}$.

Model. -We have developed a simple model that can predict the final state. We assume that this state consists of two overlapping regions: a large background, with low and uniform vorticity, and a small vortex, with high and uniform vorticity. The background in our model is centered on the trap axis, while the small vortex has a shift $D$ from the axis $(D \geq 0)$. The background has a radius of $r_{b}$, $0 \leq r_{b} \leq 1$. The strong vortex is defined by having vorticity comparable to the initial vorticity of the rings. Chen and Cross [13] have concluded from numerical simulations of a similar problem that the core region with vorticity higher than 0.7 of the initial vorticity is essentially nonmixing. Our experiments and simulations also show that a level of 0.7 of the initial vorticity is reasonable for the definition of the coherent vortex, and we use this value in our model. We also verified that the model is not sensitive to the choice of this value. With this vorticity specified, there are only four parameters that characterize the final state: the radius $r_{v}$ and off-axis shift $D$ of the small vortex, and the vorticity $n_{b}$ and the radius of the background $r_{b}$.

Possible final states of our 4 degrees of freedom model $\left(r_{v}, D, n_{b}, r_{b}\right)$ are obtained using three constraints: conservation of total circulation (charge), energy, and angular momentum. Using the scaled variables of Ref. [6], the energy is $E=\frac{1}{2} \int n \phi d^{2} \mathbf{r}$, where the potential $\phi$ is the solution for the Poisson equation $\nabla^{2} \phi=-4 \pi n$, with the boundary condition $\phi=0$ at $r=1$. The parameter $r_{v}$ determines the circulation in the strong vortex, while $n_{b}$ and $r_{b}$ define the circulation in the background. Although the loss of electrons to the walls
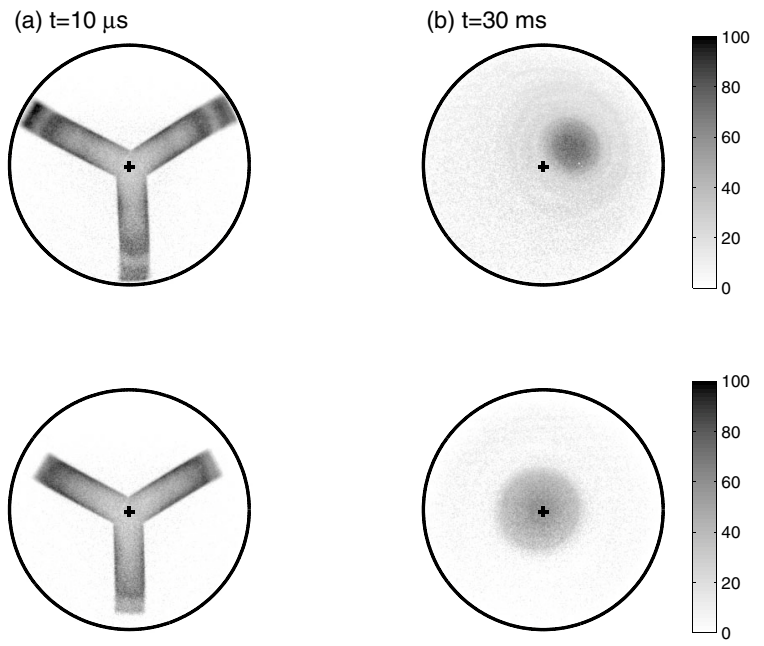

FIG. 4. Initial and final states in experiments with tribars of different sizes. occurs at longer time scales in the experiments, the transition to the metastable state occurs on a fast enough time scale such that this loss is insignificant. Our fluid simulations, which allow no loss of circulation, demonstrate that the transition to off-axis states occurs with a strict conservation of circulation.

Figure 6 parametrizes the solution as a function of the angular momentum $M$ and the off-axis shift $D$. For each initial condition (the hollow rings shown in the first three figures), we calculate the circulation, the energy $E$, and the angular momentum $M$. Then, we solve for allowed values of $D, r_{v}, n_{b}$, and $r_{b}$. As there are only three constraints for the 4 degrees of freedom, we get a onedimensional family of allowed solutions. Consequently, we arbitrarily pick a $D$ and find the allowed values for $r_{v}$, $n_{b}$, and $r_{b}$. We solve this nonlinear system using a MathCad ${ }^{\circledR}$ numerical solution finder or alternatively using an iterative search. In principle, multiple solutions are possible, but for a given $D$ we never found more than one solution with positive, real values for all the parameters. Noting that the background vorticity must fit within the system, we divide the solutions into allowed final states $\left(r_{b}<1\right)$ and forbidden states $\left(r_{b}>1\right)$, as shown in Fig. 6 . For low angular momentum the allowed range of solutions always includes an on-axis solution $(D=0)$, while for high angular momentum $(M \geq 0.3)$ states only off axis $(D>0)$ were found. As can be seen in Fig. 6, both the experimental and numerical results show that the flow evolves to the member of the one-dimensional family with the minimum allowable value of $D$, which is equivalent to the member that maximizes $r_{b}$, or equivalently the solution that maximizes the entropy. With this criterion, the preferred solutions for angular momentum smaller than $M \sim 0.3$ will be on axis $(D=0)$. At $M \sim 0.3$, the value of $r_{b}$ for the on-axis solution reaches unity. Thereafter, as $M$ increases, the preferred solutions follow

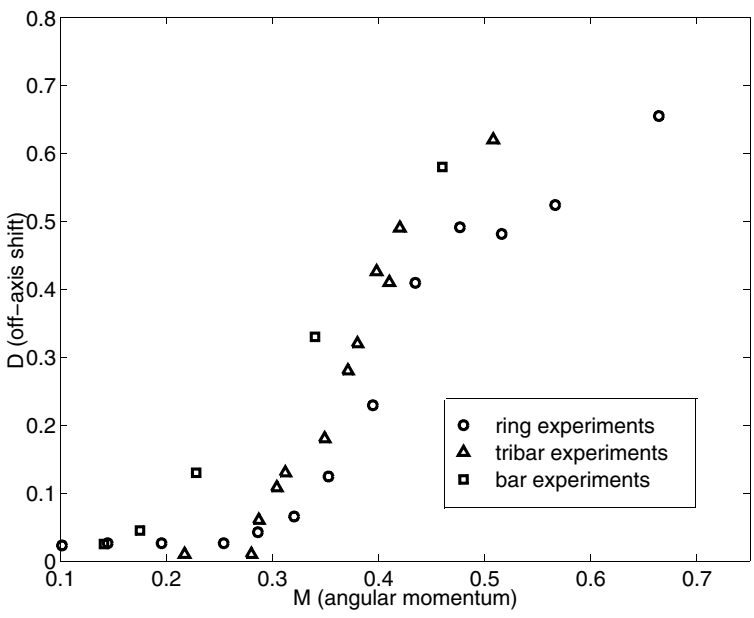

FIG. 5. Off-axis shift as a function of angular momentum for experiments with different initial electron distributions: rings, bars, and tribars. 


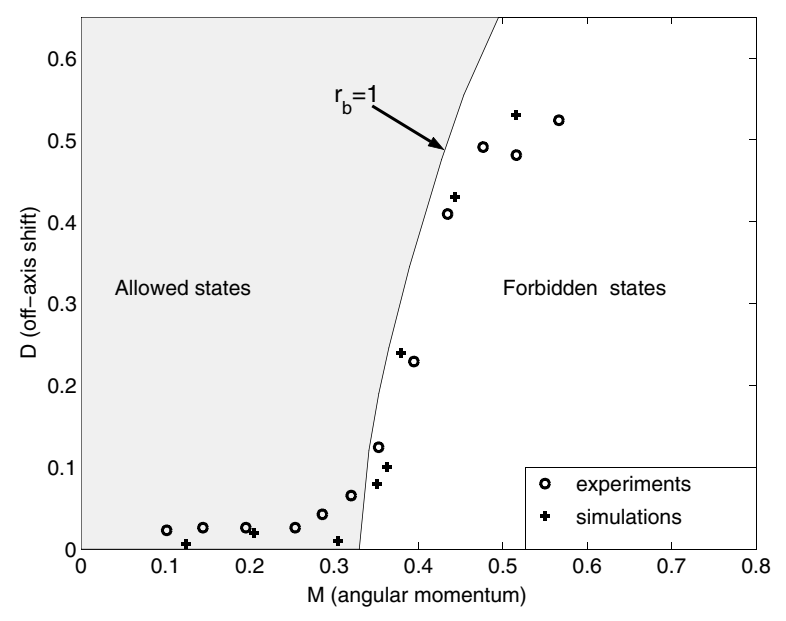

FIG. 6. Allowed solutions to the simple model for the final states, satisfying conservation of circulation, energy, and angular momentum. Also shown are the results of the experiments (circles) and simulations (crosses).

the allowed-forbidden separatrix, and $r_{b}$ remains fixed at unity. The flow is off axis; $D>0$ and increases with increasing $M$. In agreement with the experiments and simulations, the total circulation turns out to typically split nearly equally between the diffuse background and the small vortex. Similar results were obtained applying the model to configurations resembling the bar experiments we performed. As with the case of the rings, onaxis solutions were obtained as long as angular momentum could be conserved with $r_{b}<1$.

Previous work looked for single-vortex solutions of the mean-field equations of the fluid in a disk [5-7]. For highenough energy both symmetric vortices at the center and off-center vortices were found as possible solutions. Beyond the bifurcation point, the off-axis solutions had higher entropy than the on-axis states. Our results agree with the phase diagram found in Fig. 12 of Smith and O'Neil [6]. In that diagram, region 1 was defined as the region where only on-axis solutions exist, while region 3 is where off-axis solutions are found and have higher entropy. Configurations of lower angular momenta in our experiments and simulations are found in region 1 of that phase diagram, while higher angular momentum configurations are found in region 3 . For example, a ring with inner and outer radii of 0.4 and 0.5 , respectively, has $E=0.76$ and $M=0.205$, and so belongs to region 1, while a ring with inner and outer radii of 0.6 and 0.7 , respectively, has $E=0.4, M=0.425$, and is inside region 3. Note that, while the energy of the larger rings is smaller, their angular momentum is high enough to be beyond the point of bifurcation.

Conslusions. - In this Letter, we showed, for the first time, experiments, corroborated with fluid simulations, that start with initially unstable, cylindrically symmetric states and result in off-axis states. The unstable configu- rations developed quickly to metastable states of high entropy by producing a diffuse background, although the strong core did not mix and remained with vorticity close to the initial one. Previous statistical models that discussed the possibility of off-axis states assumed ergodicity [5-7]. Our results differ substantially from these models that predicted that the final off-axis configurations would be small localized columns with no extended diffuse background.

In our model we consider only a subset of all possible final states available under the constraints of conservation of circulation, angular momentum, and energy. By such restriction, we explicitly assume that ergodicity is not applicable to our system. Lack of ergodicity was also invoked by Jin and Dubin [14] to explain the creation of vortex crystals in trapped electron plasmas. Their key idea was that the low vorticity background is well mixed, while the strong vortices are not. Chen and Cross also discussed lack of ergodicity in a problem similar to ours [13], but did not apply this reasoning to their analysis of off-axis states. Our model demonstrates that maximizing entropy by way of increasing the radius of the diffuse background can lead to the sharp transition to off-axis metaequilibrium states when the radius of the background attains its maximum value $\left(r_{b}=1\right)$.

We thank Leah Zimmerman who performed some of the first experiments with large rings and Ashraf Youssef who wrote the 2D fluid simulation code. The research was supported by NSF and ONR.

[1] P.S. Marcus, Annu. Rev. Astron. Astrophys. 31, 523 (1993).

[2] D. G. Dritschel and B. Legras, Phys. Today 46, No. 3, 44 (1993).

[3] P. H. Chavanis, J. Sommeria, and R. Robert, Astrophys. J. 471, 385 (1996).

[4] J. Miller, Phys. Rev. Lett. 65, 2137 (1990); J. Miller, P. B. Weichman, and M.C. Cross, Phys. Rev. A 45, 2328 (1992); R. Robert and J. Sommeria, J. Fluid Mech. 229, 291 (1991).

[5] R. A. Smith, Phys. Rev. Lett. 63, 1479 (1989).

[6] R. A. Smith and T. A. O’Neil, Phys. Fluids B 2, 2961 (1990).

[7] P. Chen and M. C. Cross, Phys. Rev. E 54, 6356 (1996).

[8] C. F. Driscoll and K. S. Fine, Phys. Fluids B 2, 1359 (1990).

[9] D. Durkin and J. Fajans, Phys. Fluids 12, 289 (2000).

[10] B. Cluggish and C. F. Driscoll, Phys. Rev. Lett. 74, 4213 (1995).

[11] E. Sarid, E. Gilson, and J. Fajans, Phys. Rev. Lett. 89, 105002 (2002).

[12] T. Matsushima and P. S. Marcus, J. Comput. Phys. 120, 365 (1995).

[13] P. Chen and M. C. Cross, Phys. Rev. Lett. 77, 4174 (1996).

[14] D. J. Jin and D. H. E. Dubin, Phys. Rev. Lett. 80, 4434 (1998). 